\title{
ANGLICISMOS Y GALICISMOS EN LOS ORÍGENES DE LA LENGUA DEL BOXEO
}

\author{
CLARA AYUSO COLLANTES \\ UNIVERSIDAD DE COMILlaS
}

\begin{abstract}
Resumen: La lengua del boxeo se forma en España a principios del siglo XX, cuando este deporte echa a andar. Las noticias y crónicas deportivas aparecidas en los diarios de entonces -el madrileño $A B C$ y el barcelonés El Mundo Deportivo - sirven de base para elaborar el corpus de anglicismos y galicismos que entraron en el primer tercio de siglo para nombrar conceptos técnicos y específicos de este deporte. Se considera el momento de penetración de estos neologismos, su uso y pervivencia. En este primer tercio de siglo fue cuando mayor número de extranjerismos penetraron, que posteriormente irían consolidándose, adaptándose o desapareciendo.

Palabras clave: lengua del boxeo, anglicismos, galicismos, neologismos.
\end{abstract}

\section{INTRODUCCIÓN}

Aunque su práctica no fuera igual, pues tenía sus variantes o mezcla de lucha y pugilato, el boxeo es un deporte muy antiguo que ya se practicaba en Grecia y formaba parte de los Juegos Olímpicos. En Roma alcanzó cotas de gran popularidad, pero desapareció a la caída del Imperio. Sería en la primera mitad del siglo XVIII cuando la lucha de hombres a puño limpio y por dinero empezó a tener éxito en Inglaterra, si bien el boxeo tal como hoy se conoce - con el uso de guantes, el reparto de la

Title: Anglicisms and gallicisms in the origins of the language of boxing.

Abstract: The language of boxing was formed in Spain in the early twentieth century, when this sport started up. The informations and the sport chronicles featured in the newspapers at the time, the madrilian $A B C$ and The Mundo Deportivo of Barcelona, were the basis to elaborate the corpus of anglicis$\mathrm{ms}$ and gallicisms which entered of the first third of the century to designate technical and specific concepts of this sport. It is considered the moment of incursion of these neologisms, its use and survival. It was in the first third of the century when a greater number of foreign expressions entered, which would later consolidate, adapt or disappear.

Key words: language of boxing, anglicisms, gallicisms, neologisms. 
lucha en asaltos y la división de los combatientes en categorías- nace cuando en 1853 el marqués de Queensberry establece unas reglas precisas. En el XIX pasaría a Estados Unidos, donde alcanzó el nivel de profesionalización y espectáculo que lo hizo ser conocido internacionalmente e incorporado en 1904 a los Juegos Olímpicos (Cardells y Gener 2004: 41-49).

La introducción del boxeo en España se debió a marineros ingleses y franceses, y fue Barcelona la primera ciudad donde se impartieron clases de este deporte, en torno a 1875 (Sánchez García 2009: 7). Iniciándose el siglo XX, esta ciudad y Madrid contaban con alguna infraestructura, como puede observarse leyendo las noticias deportivas que esporádicamente aparecen en El Mundo Deportivo, periódico barcelonés que inicia su andadura en 1906, y $A B C$, madrileño fundado en 1903. Seguir las noticias y crónicas de boxeo que aparecen en la hemeroteca digital de ambos desde sus inicios es un modo privilegiado de asistir al nacimiento de la lengua del boxeo en España, pues en esa primera década del siglo XX está en sus primeros vagidos. En la tercera ya se puede considerar que el estirón es considerable, pues la información aumenta a tenor del creciente interés que despierta en la misma sociedad. No en vano, en 1925 Antonio Ruiz se proclama campeón de Europa de los pesos pluma y empieza a sonar Paulino Uzcudun, que tantas expectativas levantaría.

Se puede decir que es en esta tercera década cuando el lenguaje del boxeo en España toma cuerpo y figura, pues, aunque sean los sesenta y setenta los años cumbre de su popularidad y afición, la terminología y la jerga no varían. Sí que se harán muy populares gracias a la televisión, hasta el punto de penetrar algunos de sus términos y expresiones en la lengua común. Los cimientos están puestos antes de la guerra y después vendrá ya la consolidación y la integración. Como sucede en la mayoría de los deportes, que en estos principios de siglo XX empiezan a ser conocidos y practicados en España, el boxeo va fraguando su particular lenguaje en castellano gracias a las crónicas deportivas que transmiten los avatares de la competición. Cada especialidad deportiva ha de valerse de una serie de términos específicos para designar los aspectos técnicos y estratégicos de su quehacer, y muchas veces no tiene más remedio que echar mano de los vocablos de la lengua original donde nacieron o se desarrollaron. Su canal de trasmisión no es otro que el escrito, y anglicismos y galicismos penetran en la lengua española en buen número a través de los periódicos y manuales de uso, cuando no por boca de entrenadores foráneos. La lengua internacional del boxeo es el inglés, pero el francés, por razones que se verán, no le es ajeno. Leyendo las dos publicaciones elegidas se puede observar una diferencia: El Mundo Deportivo tiene tendencia al léxico francés, pues maestros franceses lo introducen en la ciudad condal, y abusa en los primeros años de él. $A B C$, por su parte, se mantiene más fiel al lenguaje internacional. Ambos periódicos, en las grandes confrontaciones 
de aquellos años —en Estados Unidos, Inglaterra, Australia, Francia, etc. — se sirven de crónicas internacionales que han de traducir. Excelsior, el primer diario especializado en deportes, nacido en 1923 en Bilbao, contribuirá también a fijar y desarrollar lo que con el tiempo ha de ser el léxico propio del boxeo en castellano.

Aunque muchos de estos extranjerismos están recogidos en diccionarios deportivos (Agulló 2003; Castañón 2004) o en diccionarios y estudios de anglicismos (Alfaro 1970; Fernández García 1972; Pratt 1980; Lorenzo 1996; Rodríguez González y Lillo 1997; Rodríguez González 2012) y galicismos (Curell 2009), de lo que se trata en este trabajo es de determinar cómo empezaron a penetrar los préstamos extranjeros de este deporte en España en el primer tercio del pasado siglo, así como su grado de aceptación y adaptación ${ }^{1}$. Como sucedió con otros deportes, ellos serán fuente de neologismos en español que no solo dan color a la lengua sectorial especializada del boxeo, sino que algunos incluso se incorporarán, con derivaciones y con sentidos figurados, a la lengua común, enriqueciéndola (Castañón et al. 2005).

\section{ANGLICISMOS}

\subsection{El nombre del deporte y su práctica}

La palabra boxeo y sus derivados aparecen en España en las últimas décadas del siglo XIX, provenientes del deporte que en Inglaterra se había hecho tan popular. To box era el verbo, que significaba 'golpear', y en este caso 'golpear con los puños'; boxing era el acto de boxear y boxer el practicante de este deporte. En el madrileño $A B C$, salvo alguna rara excepción en que aparecen box y boxe, lo habitual en las primeras décadas del siglo XX es denominarlo boxeo, al contrario de lo que ocurre en el barcelonés El Mundo Deportivo, que emplea habitualmente boxe (en femenino, la boxe), aunque boxear y boxeador. Ello tiene su explicación: fueron maestros franceses los que introdujeron el boxeo en Barcelona, lo que hace que en esos primeros años se note en algunos otros términos. Esta denominación de boxe no desaparecería en el periódico hasta la década de los treinta: "La boxe de Arambillet, esa boxe que en el campo le dio justa fama de primerísima figura, apareció ayer en el ring del Prince con todo su esplendor" (MD 7-III-1938: 1). A principios de siglo también se refieren a este deporte como la lucha de boxeo y se llama luchadores a los que se

\footnotetext{
${ }^{1}$ Aunque las conclusiones de este trabajo no coincidan exactamente con lo que a rasgos generales determinan para la introducción y consolidación de anglicismos en España, interesa leer Rodríguez González (2012: 320 y ss.) y Vázquez-Amador y Lario de Oñate (2015).
} 
enfrentan: "El premio era de 100.000 francos, y los luchadores, Carpentier, francés, y Klaus, americano, ambos famosos campeones" ( $A B C$ 25-VI-1912: 5). El término púgil, como alternativa de boxeador, aparece en la segunda década, junto a otros de su familia como pugilismo y pugilista. Esta última denominación tanto se usa como adjetivo — aplicándose a match, velada, campeón, etc. - como sustantivo: "el notable pugilista Mc Klosky, que ha venido a Barcelona para participar en los festivales del Frontón" (MD 14-XI-1916: 4), sin duda calcándolo del inglés pugilist, muy utilizado allí, como en Francia pugiliste. Puglístico (pugilistic) vendría en la década siguiente: "Larruy, que conoce a fondo y prácticamente el valor pugilístico de muchos boxeadores, para dar una «gran velada »" (MD 13-X-1921: 4),

En el capítulo dedicado a este deporte en su libro, Antonio Viada (1903: 685705) recoge, junto a boxeo, otros términos tomados del inglés, bien de forma directa, tal cual: boxers, ring y boxing-ring, rounds, knockout, punch ball y fight, bien en traducciones como guardia, paradas, árbitro y pesos, y da dos mal dobladas: manager la hace equivaler a 'cuidador' y referee a 'voceador', lo cual quiere decir que los conocimientos del boxeo inglés en España eran titubeantes y no estaban en absoluto fijados. Lo irán fijando los cronistas deportivos en la prensa a medida que corre el tiempo. El enfrentamiento o combate entre púgiles se denominaba preferentemente match, a veces sucintamente anunciado: "Después de catorce asaltos, la Policía suspendió el «match», y el Jurado declaró vencedor a Johnson” ( $A B C$ 1-I-1907: 7), ganándole esta palabra francesa el terreno a otras inglesas como contest o fight. Combate, otro galicismo adoptado por el inglés — combat - será una alternativa que aparece también muy pronto: "El combate entre el campeón mundial en boxeo, Tommy Burns, y el negro Jack Johnson ha sido un acontecimiento deportivo sensacional que ha llevado a Nueva York a miles de curiosos" ( $A B C$ 1-I-1909: 7). El fight inglés que copia Viada (1903: 702) enseguida se traduce por lucha o pelea, que, junto con encuentro, serán sinónimos de menos uso. Sí que se recurrió en la tercera y cuarta década del siglo al derivado in-fighting (infighting) para nombrar la mayor cercanía en la lucha de los contendientes, pero desde entonces se olvidó a favor del calco francés cuerpo a cuerpo. Muy temprano y de manera aislada aparecería en $M D$, con una explicación suficiente en la que se dice que es método americano: "arte de combatir y pegar muy fuerte en los cuerpo a cuerpo" (MD 18-II-1909: 3). Para nada, en cambio, aparece en la prensa hispana su antónimo inglés outfighting, que señala el mantenimiento de la distancia larga. 


\subsection{El derribo del contrario}

Desde un principio también alternan roundy asalto, prevaleciendo mucho más el primero, sobre todo en $M D$, que en ocasiones también recoge el término francés reprise. A veces se citan los dos, anglicismo y traducción: "El negro Johnson ha vencido al boxeador blanco Jeffriers. Este quedó fuera de combate en el decimoquinto round (asalto)" ( $A B C$ 6-VII-1910: 14). Ring es una palabra que se impone de manera casi absoluta desde el principio de siglo, pues solo tímidos sustitutos como tablado o tapiz aparecen en la segunda década. Tapiz comienza a hacerse habitual iniciándose los treinta, lo mismo que lona, ambos como metonimias de ring. La auténtica alternativa de este, cuadrilátero, aparece en 1927: "El cuadrilátero estaba espléndidamente iluminado por 40 focos, que arrojaban un verdadero torrente de luz sobre los pugilistas" ( $A B C$ 22-VII-1927: 20). A partir de ese año se hace habitual hasta que a mediados de siglo se le equipara. Este término le ganó la batalla a cuadrángulo, pues así se le decía también por entonces: "se había librado a los luchadores (de la fina lluvia) por medio de un dosel colocado sobre el cuadrángulo" (E 9-III1927: 1). Corner se llamó al principio cada esquina, como en los campos de fútbol, aunque acabaría triunfando rincón. A principios de los años treinta aparece ringside para nombrar los asientos preferentes, en las primeras filas en torno al ring: "Entre el público de ringside hay un momento de gran expectación, ya que se prevé la caída del alemán" (MD 13-VI-1930: 3). Su uso, sin embargo, ha sido escaso e irregular en adelante, refloreciendo a finales del siglo XX.

Desde muy pronto hizo también fortuna knock-out (knockout): "Kubiak logró una brillante victoria, consiguiendo el knock-out al décimo round" (MD 4-XI1909: 4), aunque se simultaneaba con el calco del francés que resultaría definitivo: fuera de combate: "Cayó k.o de un golpe de crochet derecho precedido de un ligero directo izquierdo [...], quedó fuera de combate irremisiblemente" (MD 2-XII1920: 4). Como se ve, el anglicismo encontraría la sigla providencial K.O. (k.o, con minúscula, se escribió también), de enorme éxito a partir de 1919. Más tarde se denominaría Knock-out técnico al episodio en que el árbitro decide dar por acabado el combate cuando estima que uno de los púgiles ha sufrido un importante castigo y seguir en la lucha le sería perjudicial, lo cual se transforma en K.O técnico (escrito a veces K.O.T) o victoria por inferioridad del contrario. En todo caso, se denomina $k a o$, por semiadaptación gráfica, al nuevo neologismo. El anglicismo puro, sin embargo, empezó por los años veinte a ser escrito en ambos periódicos como se pronunciaba en inglés: nocaut, y así se iría dando paulatinamente hasta nuestros días, forma que la RAE (2014) recomienda especialmente. La transformación se realizó en una entrevista hecha al primer campeón de Europa que tuvo España. Merece la 
pena la conversación:

La única vez en su vida que quedó...

— ¿Cómo se dice, Ruiz? ¿Knock-out?

-Sí, señor: nocaut; pero las palabras no tienen importancia. Se escribe K.o.

Apúntelo usted. Pero no haga caso; eso son costumbres de este deporte.

- ¿Qué se siente cuando queda uno conmocionado?

-Eso es: conmocionado... Pues se ven luces y se oyen muchas campanas.

(ByN 15-XI-1925: 77).

Por estas mismas fechas de la segunda mitad de los años veinte, en pleno apogeo del vasco Uzcudun, tan proclive a vencer a sus adversarios por K.O., esta expresión inglesa produce un verbo en castellano, el verbo noquear, que se hace habitual a partir de los años treinta, pasando a formar por derecho propio parte de nuestro idioma: "y el entusiasmo que produjo Paulino al noquear tan limpia y contundentemente a Harr, fue desbordante" ( $A B C$ 8-VIII-1927: 11).

Para anunciar el derribo de un púgil, que, sin embargo, se levanta antes de la cuenta fatídica de diez segundos y reanuda la pelea, está el knock down, que aparece en la segunda década tímidamente y, aunque nunca se pierda del todo, no se prodigará mucho en las crónicas: "Kid está ya desorientado, pega un directo y recibe un croche en la mandíbula que le hace caer «knock-down», no levantándose hasta que el árbitro contó siete" (MD 15-V-1916: 4). Anglicismo mucho menos usual que el anterior, su significado se dice mejor en español a través de circunloquios o locuciones del tenor de irse a tierra, ser enviado al suelo, ser derribado, besar el tapiz (la lona, elpolvo) ${ }^{2}$. Popular, en cambio, se ha hecho en castellano groggy, que hizo su aparición iniciándose el tercer decenio del siglo para expresar el estado tambaleante y de aturdimiento sobre el ring de un púgil que ha sufrido un duro castigo en la pelea: "en el primer y segundo round el dominio de Alís fue grande, tanto que al primer round sufrió un k. d. y estuvo groggy unos momentos” (MD 8-IX-1921: 6). Muy pronto, este anglicismo apareció adaptado a la grafía castellana como grogui, pero sin continuidad, solo de forma esporádica: "Mompó tuvo «grogui» varias veces a su adversario" ( $A B C$ 17-XI-1926: 35). Ha sido finalizando el siglo XX cuando ha empezado a regularizarse esta forma.

2 Según Fernández García (1972: 151-152), este término se utiliza en inglés en la lucha libre, pero no en el boxeo. En España, sin embargo, apareció en las crónicas de boxeo mucho antes de 1927, que es cuando él lo fecha por vez primera. 


\subsection{Pesos o categorías}

Según el peso, los boxeadores se dividen en distintas categorías para evitar que haya diferencias considerables de volumen y fuerza en el enfrentamiento. Estas categorías han ido perfilándose a lo largo del tiempo. Iniciado el siglo XX, Viada (1903) recoge en su libro cuatro peso de pluma, peso ligero, peso medio y peso gordo. En 1917 en $A B C$ ya se leen hasta ocho, enunciados así: moscas, batam (sic), pluma, ligeros, walter (sic), medios, medio-pesados y pesados. El peso menor acogía a los que no llegaban a 50,30 kg, y el mayor a los que pasaban de 79,25 kg. Hoy, siguiendo a la AIBA (Asociación Internacional de Boxeo Amateur), las categorías para los boxeadores amateurs son once, pues se han recompuesto las diferencias añadiendo por abajo el peso mini o semi-mosca (hasta $48 \mathrm{~kg}$ ), por arriba el super pesado (más de $91 \mathrm{~kg}$ ) y entre medias el superligero o wélter junior (hasta $64 \mathrm{~kg}$ ). El nombre en castellano está, poids lourds, calcado del original inglés, pues son auténticos calcos léxicos que traspasan de aquella lengua el significado adaptándolo a la colocación propia. Así tenemos peso semi-mosca / light flyweight; peso mosca / flyweight; peso gallo / bantamweight; peso pluma / featherweight; peso ligero / lightweight; peso wélter / welterweight; peso medio / middleweight; peso semi-pesado / light heavyweight; peso pesado / heavyweight y peso superpesado / super heavyweight. Las categorías oficiales para los profesionales suben a diecisiete, añadiendo por arriba el peso crucero / cruiserweight y por abajo el peso minimo (peso paja) / minimumweight.

Aunque en menor escala, el peso medio siempre se ha dicho también peso mediano: "El púgil español se ganó el derecho a disputar el título mundial de peso mediano frente al alemán Félix Sturm” ( $A B C$ 14-XI-2007: 98). En cuanto al que terminó llamándose peso pesado, hubo al principio distintas vacilaciones. Se llamó pesos grandes, y semi-grandes al en un principio medio-pesado: "París, 25, 8 mañana. Campeonato mundial de boxeo, pesos semi-grandes" ( $A B C$ 26-IX-1922: 26), y también pesos fuertes, e incluso pesos gordos. Es a partir de los años treinta cuando se unifica en lo que ahora se conoce, combinándolo a veces con pesos máximos, como siempre sucedió: "hace tiempo que Ernie Schaaf es uno de los tres primeros boxeadores de la división de los pesos máximos" ( $A B C$ 21-IV-1932: 48). En la adopción definitiva debió de influir la denominación francesa poids lourds.

Del mismo modo, el peso gallo se denominó así por influencia del francés poids

${ }^{3}$ Como explica Alfaro (1970: 99), bantam es el nombre de una gallinácea muy pequeña que se cree originaria de Java, pero que, tanto este como welter, son nombres propios intraducibles. E. Lorenzo (1996: 471) dice, sin embargo, que welter significa "tumulto, maremágnum" en inglés, pero que existe también el verbo to welt, entre cuyas acepciones está la de "golpear". 
cocq, y $M D$ lo adoptó desde un principio, no así $A B C$, que eligió la denominación inglesa bantam weight (no sin distintas vacilaciones: "batam", "bantán”, "battam”...) y la fue utilizando hasta 1972 en distintas ocasiones, si bien hay que decir que, a partir de 1922, en que introdujo la traducción del francés, ha hecho a esta última mayoritaria. Este es el anuncio en el periódico de dos veladas distintas: "Pesos bantam. - J. Vernacci, F. Rivero, R. Sáenz de Heredia, F. González” ( $A B C$ 20-I-1918:15) y “1. Dorado-Luque, pesos gallos. Cuatro asaltos de dos minutos" (ABC 7-V-1922: 32) .

\subsection{La pegada y los golpes}

Capítulo aparte merecen los golpes de boxeo, cuya diversidad y grado es de conocimiento propio de especialistas y aficionados. Punch es palabra inglesa que significa 'vigor, fuerza, potencia', y también 'puñetazo' o 'golpe dado con el puño cerrado' y que aparece en la segunda década del siglo XX con el sentido de fuerza y contundencia del golpe: "Bien diferentes son los estilos o medios que ambos boxeadores tienen; el uno es dueño de un «punch» terrible que no perdona nada cuando consigue asestar un buen golpe" (MD 16-I-1913, 4). En los años veinte se hace ya habitual (en $A B C$ no se utiliza hasta 1923) y en este tiempo se le busca también una palabra ajustada en español, que será pegada: "si a su vez no logró poner jamás en peligro a Paulino, cúlpese de ello a lo poco eficaz de su pegada” (MD 4-VIII-1924: 4). Será esta, sin duda, un auténtico neologismo interno que, desde entonces, se incorpora al castellano. Existen varios compuestos sintagmáticos con la palabra inglesa, como rabbit punch, que se traduce como golpe de conejo y que algunas, pocas veces, se ha escrito: "no debió consentir el «rabbit punch» de Arteche a Rhor. El golpe en la nuca es permitido cuando los pugilistas se encuentran frente a frente. Jamás cuando el que lo recibe se encuentra de espaldas" (E 27-III-1927: 1). Y también punch drunk, calificativo que se aplica a un boxeador cuyo cerebro se ha visto afectado por el castigo de los puños, tampoco es muy corriente, pero se da: "Y hasta los «punch drunk $\gg$ - púgiles sonados- que mendigan una bolsa para arrastrar pesadas glorias sobre la lona" ( $A B C$ 23-III-1977: 33). Algo más se recoge la expresión inglesa lucky punch, traducido por golpe de suerte: "Cuando un «experto» español en boxeo escribe «lucky punch» en lugar de «golpe de suerte», suponemos amablemente que debe tener sus razones" ( $A B C$ 31-VII-1927: 29).

Emparentadas con la palabra inglesa están otros dos términos que se incorporan a la jerga pugilística en los gimnasios: punching ball y punching bag. El primero (que también se ha escrito con solo su primer término punching) sería una especie de balón colgante con el que los pugilistas se entrenan, y aparece muy pronto: "Una nueva mejora acaba de implantarse en el Gimnasio Vila. Desde primeros de este 
mes, funciona un soberbio Punching-Ball, así como el saco de arena indispensable para un verdadero entrenamiento de la Boxe inglesa" (MD 11-XI-1909: 4). Se ha traducido como balón de golpeo (o pegar), así como el segundo por saco de golpeo (o de pegar), y más coloquialmente entre los ejercitantes del deporte: balón o pera y saco, respectivamente. Estas formas, sin embargo, serían falsos anglicismos, deformaciones de punch-ball y punch-bag (Rodríguez González 2013: 169), como no es anglicismo puncheur, aplicado al púgil de gran pegada, porque esa forma es la versión francesa del puncher inglés, y que asoma en los veinte, haciendo fortuna en adelante para caracterizar a los también llamados golpeadores y pegadores, dispuestos a vencer por la vía rápida: "pero ante Sáez, le pudimos apreciar una gran esgrima, que decía claramente que no solo era un «puncheur» sino un excelente boxeador” (MD 4-I-1924: 4) $)^{4}$.

De counterpunch se formaron también contragolpe y contragolpear: "Latzo contragolpeó con su izquierda a la quijada, que es lo que le convenía, ya que Dundee no es duro de maxilares" (MD 6-VII-1927: 1), y, naturalmente, en segunda instancia contragolpeador, nombre con el que se conoce al boxeador que está a la expectativa del ataque de su rival para bloquearle y asestarle un golpe a la contra.

Golpe, puñetazo e, incluso, disparo traducen en castellano voces inglesas como strike, blow o shot, de significado genérico, porque luego las diversas formas de golpeo dan lugar a una serie de hipónimos que, en buena parte, han pasado sin más al español. El jab es un golpe en corto que se realiza con la mano que se tiene adelantada en la defensa - la izquierda en los diestros - y se utiliza mucho, tanto para mantener al contrario a distancia como para despistarle mientras se prepara otro golpe más contundente con la otra mano. Su nombre inglés, sin embargo, no fue de los que primero penetraron en las crónicas, pues no se hace familiar sino a partir de la tercera década, ya que aparece un poco antes: "Soum pegó un buen jab izquierdo, seguido de un crochet a la cara” (MD 5-II-1918: 4). Jab significa algo así como 'punzada' y en castellano se utilizan entre el gremio voces como punteo y picotazo.

Mucho más usados en las crónicas fueron el uppercut y el swing, que aparecen también en la primera década. El primero (a veces escrito upercut) es un golpe que se dirige de abajo arriba al mentón del oponente: "Johnson aplicó un terrible «uppercut» del derecho sobre la mandíbula, rodando Burns por el suelo" (MD 18II-1909: 3). El segundo es un golpe largo en el que se gira el puño para golpear con los nudillos: "Johnson logró acorralarlo en un rincón, y le descargó sucesivamente

${ }^{4}$ Curell Aguilá (2009: 422) dice que esta palabra se incorporó al francés en 1923, por lo que en España se tomó de inmediato. 
tres formidables «swings»" (MD 26-II-1914: 4). También se llama bolea: "Hilario larga un derechazo a la cara de Dundée, quien responde con una bolea floja" ( $A B C$ 8-VII-1928: 29). Un golpe lateral con el brazo doblado, que se dirige a la cabeza o a los riñones, es el que generalmente aparece como crochet, nombre francés que significa "gancho", ya que sigue una trayectoria semicircular. Será muy utilizado, y aparece muy pronto: "le alcanzó con un crochet con la mano derecha, de vertiginosa velocidad, a la punta de la barbilla" ( $A B C$ 14-III-1912: 25). Traducido como gancho al castellano, es una segunda opción para referirse a este golpe, aunque más tardía: "El guipuzcoano puso fuera de combate a la «Pantera » de un rápido y potente gancho con la derecha" ( $A B C$ 18-IX-1928: 18). Y una tercera opción, poco usada pero existente a lo largo del tiempo, es el hook inglés, con el mismo significado: "Bautista fue vencido, al tercer round, como consecuencia de un hook que propinó Hernández y que dio en tierra con el vencido" (MD 19-II-1920: 2).

Un golpe muy utilizado es el cross o directo, similar al jab pero ejecutado con la mano contraria, que sale desde atrás y por eso lleva más potencia; además se utiliza a la contra, para frenar al púgil que ataca. El término inglés aparece muy pronto: "En el cuarto round, Camille recibió un formidable cross bajo la nuca" ( $A B C$ 26VI-1911: 13), pero, aunque seguirá siempre vigente, su uso es sobrepasado por el directo español, traducción calcada del straight o right, nombres que también recibe en inglés, o del direct francés. Empieza a utilizarse también pronto: "Respóndele Pomés con buenos directos y a estos Hoche con un crochet superiorísimo" (MD 8-XI-1915: 4). Y también ha sido llamado recto y, en esos primeros decenios derecho, sin que cuajasen, como sí lo hizo cruzado, traducción del primer término inglés nombrado.

Aún habría que añadir otros dos golpes de nombre inglés: el sacacorchos, calco del inglés corkscrew, que aparece escasamente citado, y el llamado bolo-punch, de formación híbrida, un particular uppercut de derecha que se hace cogiendo mucha curva. Entre los españoles lo practicaron mediado el siglo Kid Gavilán y Pedro Carrasco. En la crónica de un combate de este se lee: "Llegó a hacer una perfecta demostración de lo que es el «bolo-punch», golpe que posee más espectacularidad que eficacia” ( $A B C$ 30-IV-1967: 109).

En los años veinte, a propósito de Dempsey, un púgil norteamericano que no daba tregua a sus contrincantes, se puso de moda la palabra killer, entresacada del compuesto killer instinct: "Dempsey es un killer; es una «fiera». Embiste más que ataca” (Miquelarena 1934: 54) y luego estaría aletargada hasta los sesenta. En los últimos años ha renacido y hoy se prodiga para calificar a cualquier deportista con ansias de victoria, atacante nato, ya sea delantero de fútbol, ciclista, etc. 


\subsection{La defensa y otros anglicismos efímeros}

Las posiciones o movimientos de defensa aportan más anglicismos que refieren otros aspectos técnicos o estrategias de combate. Muy socorrido es el clinch, que aparece en la segunda década para designar la acción en que un púgil agarra o bloquea los brazos de su oponente para evitar su ataque mientras toma resuello: "Empieza el combate sin referée y naturalmente al primer «clinch» hay bronca en el público y alguna zancadilla en los maestros" (MD 4-X-1915: 3). En la jerga interna se conoce también como cincha, grapa u horquilla (Rodríguez Feu 1987: 84-85). Crouch designa un particular modo de defensa, que consiste en mantener los puños altos y pegados a la cara. Apareció en las tercera y cuarta décadas, pero en adelante no cuajó: "Lany abusó del «crouch», posición que lo reservaba algo de las cruces o ganchos de Terry" (E 1-VI-1927: 1). También side step tuvo una fugaz aparición en el segundo cuarto de siglo para indicar el desplazamiento con que eludir un golpe: "la alegría corrió a cargo de Ara, con sus «side stops», su compostura de «arlote» y su boxeo efectista" (E 15-V-1927: 2). En cuanto a feint, que alguna vez aparece, pronto es sustituido por finta: "El primer round fue de estudio. Allak inició muchas «feintes», pero sin entrar” (MD 22-X-1914: 3).

Otros anglicismos sin mucho éxito, de corto alcance en uso y años en las crónicas, fueron términos como reach, empleado para designar la capacidad de alcance del brazo de un púgil: "Además lleva la ventaja de su reach y de su mayor peso y creo que sacando provecho de esto podrá vencer en forma categórica” (MD 22-IV-1929: 7); rush (roush en otras ocasiones) para aludir a la agresividad o apretón final de los púgiles, aunque era término que antes se había aplicado al ciclismo y a otros deportes de velocidad: "Campo se lanza a un rush intentando ganar con un solo golpe, e Hilario cometió la imprudencia inexcusable de aceptar ese género de pelea” ( $M D$ 26-II-1926: 2); y lo mismo sucedía con fould (también foul), por falta o acción antirreglamentaria, tomada del fútbol u otros deportes colectivos: "El boxeador español Ferrand fue proclamado vencedor sobre Phil Tobías, al cometer este un «fould» en el segundo asalto de su combate" ( $A B C$ 5-III-1930: 38). Raro también es timing, que aparece alguna vez, aunque viene a significar algo que hoy se usa mucho, sobre todo en política, y es 'saber jugar con los tiempos' o 'marcar los tiempos'. Hablando el cronista de Paulino Uzcudun dice que "acierta admirablemente a hacer lo que llamamos el «timing», cosa que no ocurre cuando dirige el fuego a la cara” (MD 26-VIII-1927: 1). 


\subsection{Roles en la pelea}

Sí que han tenido éxito otros anglicismos como sparring y challenger, que se dieron a conocer al comienzo de los años veinte. El primero lo hacía en la forma compuesta inglesa de sparring-partner ('compañero de entrenamiento'), que se repitió mucho alternándose con la simple, que es la que acabó imponiéndose: "El día 10 tendrá lugar su exhibición con su sparring partner Lenaers, reproduciendo ante el público sus famosos combates con Beket y Battling Levinsky" (MD 17-III-1921: 4). A challenger - que Rodríguez González (1913: 143-144) considera seudoanglicismo porque no existía en esa lengua como tal, sino que es una derivación de challenge 'prueba de alta competición, que se hace a propósito'- se le dio desde el principio el significado de aspirante al título o retador del campeón: "En Nueva York se ha celebrado un combate entre el campeón del mundo de pesos ligeros Benny Lonard y Joe Willíng, «Challenger» al título" (MD 9-XII-1920: 4). La traducción más exacta y repetida es la de aspirante, con opciones sinonímicas como candidato o pretendiente (al título).

Otros anglicismos empleados para señalar personas relacionadas con este deporte han sido referée, manager y speaker. Los tres son compartidos con otros y tienen pronta presencia en las crónicas. El primero se venía ya usando a principios de siglo para el fútbol y se utilizó combinándolo con su traducción, árbitro, durante mucho tiempo, aunque a partir de los cuarenta cada vez aparece más esporádicamente. Lo mismo sucede con manager para indicar al preparador o director deportivo del púgil, que con el tiempo se ha singularizado como el que le promociona y administra su vida profesional. El speaker forma parte de la mesa técnica del combate y es el que anuncia a los púgiles al principio y el resultado al final: "El público armó un escándalo formidable cuando el «speaker» anunció la descalificación de Carpentier, por haber invadido el «ring» su «manager»" (ABC 28-VI-1912: 3). Para atender y aconsejar al púgil en los descansos entre asaltos, está el segundo, que se toma del second inglés, y su auxiliar. El árbitro anuncia el comienzo de cada asalto con el clásico "Seconds, out". Para nombrar al organizador de combates y veladas se utilizó el nombre de promotor, tomado del francés desde muy pronto, como se deduce de esta cita: "pero como dicho encuentro fue concertado cuando se creía a aquél desposeído de su título y los gastos de los «promoteurs» de dicho encuentro, ya estaban hechos" (MD 25-XII-1913: 5), pero a mediados de los años veinte hizo su aparición la voz compuesta inglesa match-maker: "El contrato para este encuentro fue firmado ayer tarde en las oficinas del Garden entre Jess McMahon, «match-maker» de la gran Corporación que preside Tex, y el gabinete en pleno" (ABC 31-VII-1927: 29). Salvo un corto tiempo, esta palabra no tendría éxito. 


\subsection{Otras expresiones y modismos}

Gong es una creación onomatopéyica malaya que, a través del inglés, pasó al francés y al castellano para indicar el instrumento que señalaba el principio y el final de los asaltos (Agulló 2003: 278). Se incorpora pronto, en el primer decenio: "Por un momento se creyó que Burns iba a quedar tendido en el suelo, pero el «gong» le salvó una vez más" (MD 18-II-1909: 3). Aunque seguiría bajo esta forma repitiéndose con regularidad, en el tercer decenio empieza a aparecer mejor adaptado a la fonética hispana como gongo: "le pegó de ambas manos en las cuerdas y fueron tantas las aclamaciones, que no fue oída la señal del gongo” (MD 9-VI-1921: 4), la cual se repetiría, si bien intermitentemente, en adelante.

Finalmente, estarían las voces que el árbitro pronuncia en cada combate y que son ya de reconocimiento internacional. Son estas: ¡Time!, que le dice al cronometrador para poner en marcha el reloj; ;Seconds, out!, para que dejen expedito el cuadrilátero los auxiliares del boxeador; ;Stop! cuando el combate debe suspenderse y ¡Box!, cuando debe reanudarse; ¡Break!, para que los púgiles que están agarrándose se separen: "Durante los tres rounds, el árbitro repitió un centenar de veces el break" (MD 20-VII-1923: 3) y iOut!, para anunciar el fuera de combate por K.O. o inferioridad de un combatiente: "Este se incorporó en el preciso instante de ser declarado «out»” (MD 28-XI-1926: 2). Y cuando en un combate el árbitro considera que no hay verdadera lucha entre los púgiles, que no hay competitividad, puede suspenderlo y declararlo no contest: "Mazzinghi — combate no contest - retiene el título" ( $A B C$ 26-X-1968: 65).

En cuanto a locuciones o modismos boxísticos importados, pueden considerarse dos: saved by the bell / salvado por la campana y throw in the towell / arrojar (tirar, lanzar) la toalla. La primera aparece antes con la variante del "gong" o "gongo": "un derecho de Criqui a la mandíbula abate a Wyns, pero este con un coraje inaudito se levanta a los nueve segundos y el gongo le salva" (MD 10-VII-1922: 1), aunque terminaría por fijarse la americana: "Al terminar varios asaltos parecía que Tito iba a caer al suelo, pero siempre fue salvado por la campana" ( $A B C$ 24-IX-1930: 33). En cuanto a la segunda, hay que decir que en el reglamento se leía: "La esponja o toalla debe ser lanzada a los pies del árbitro o director de combate para que este se dé cuenta de la misma" (MD 23-VIII-1936: 3). En la primera mitad del siglo se usó de manera general la locución que se refería a la esponja: "Francis Charles vence a Eustache fácilmente, pues al empezar el tercer round el cuidador de Eustache tiró la esponja e hizo bien" (MD 8-V-1919: 4), porque al cruzar la segunda mitad, esta desaparece y se impone la referente a la toalla, que apenas había aparecido anteriormente de manera aislada: "la medida de que se trata tiene su origen en las reclamaciones 
que se produjeron con motivo del «match» Tod Morgan-Kid Sullivan, en que el «manager» de este último arrojó la toalla al «ring»" ( $A B C$ 2-IX-1926: 33). Ello hace pensar que la locución anglicista se impuso, aunque tardíamente, a la francesa, pues la acuñación propia de esta lengua es la de jeter l'éponge.

\section{GALICISMOS}

Falsos anglicismos introducidos en castellano del francés, que los creó sobre términos ingleses, son footing y forcing. El primero es una carrera suave y continuada que los boxeadores realizan en sus entrenamientos, tomado de to go to foot. El segundo, del verbo to force, se utiliza en Francia desde 1912 (Agulló 2003), y al año siguiente ya estaba en España: "Grande fue el número de personas que acudió a presenciar el encuentro, abundando en gran manera el forcing en el cual Klaus dominó a Papke" (MD 13-III-1913: 5). Forcing es el ataque redoblado o furioso.

Estos dos, junto con las también adaptaciones francesas del inglés challenger, puncheur, crochet..., no serían sino uno de tantos galicismos que se han colado en el boxeo en castellano. Un importante número de vocablos ingleses proceden de la lengua francesa de la esgrima, que los tomó prestados para denominar operaciones similares en la lucha con los puños. La esgrima o lucha a espada es algo antiguo, que ya se practicaba a su modo en la Edad Media. En el Siglo de Oro estuvo de moda en España, de donde pasó a Francia, en cuyo siglo XVIII fue debidamente regulado con la consiguiente forja de un lenguaje que se extendería a Europa. Los aristócratas ingleses en los siglos XVIII y XIX prefieren dirimir sus duelos con los puños en vez de con armas, por lo que el boxeo incorpora términos propios de la esgrima, en su original francés, adaptándolos, y los trasmite de segunda mano al español (Cebrián 1989: 106). Así, las palabras ya vistas de combat y match, que pasan tal cual al inglés 5 . Match era en la esgrima el cara a cara entre los dos contendientes buscando tocarse con la punta del florete para marcar puntos. El empate se denominaba match nule, y así pasó al inglés, mientras que el castellano optó por hacer la composición híbrida match nulo: "Match de boxeo: míster Charles Harper y monsieur Víctor Keilhaner; match nulo (empate)" (ABC 20, IX-1908: 4). Y avant match se denomina en la jerga boxística a los momentos precedentes al combate, también en español.

Otros sustantivos que pasan de la esgrima al boxeo, con origen francés, adopción inglesa y posterior castellana son assaut / assault / asalto; contact / contact / contacto; attaque / attack / ataque; contre-attaque / counter-attack / contraataque;

5 El francés sería el “étimo último”, no el “inmediato”, según Pratt (1980: 36). 
offensive / offensive / ofensiva; contre-offensive / counter-offensive / contraofensiva. Entre los golpes, están estos: contre / counter / contra; direct / direct / directo; double / double / doble; une-deux / one-two / uno-dos: "González se impone: coloca los golpes más varios, y sobre todo algunos «uno-dos» plenamente logrados, que el público aplaude mucho" (MD 25-V-1923: 3). Referencias a la defensa hay muchas: défense / defense / defensa; déplacement / displacement / desplazamiento; distance / distance / distancia; esquive / esquive / esquiva: "esquivar la acometida de la fiera no es sino la esquiva del boxeo o el dribling o el regate del fútbol: una retirada suficiente para evitar el ataque del contrario" (ByN 31-X-1926: 75); feinte / feint / finta; garde / guard/ guardia; parade / parade / parada; riposte / riposte / respuesta: "y así como en la esgrima existe la frase parada y respuesta, así un golpe en la «boxe» debe pararse, atento siempre a responder con un ataque" (ABC 29-VIII-1926: 39) ${ }^{6}$. Y coquille / coquille / coquilla, el protector de la pelvis que, alguna vez, aparece en francés: "Entre las cosas que no merecen nuestro aplauso, antes al contrario, puede clasificarse la ausencia de «coquille» con que muchos de los amateurs presentáronse al ring" (MD 15X-1924: 2), aunque en adelante se adapte. También son de uso verbos como armer (un coup) / to arm / armar; désarmer (la garde contraire) / to desarm / desarmar; se couver / to cover one self / cubrirse; toucher / to touch / tocar; redoubler ( 1 'attaque) / to redouble / redoblar; manoeuvrer / to maneuvre / maniobrar; contrer / to counter / contrar, particular traducción que desplaza a 'contrarrestar', solo en este deporte: “Gray posee un formidable derecho (sic) que parte directamente sin finta alguna y al cual hubiese podido contrar Miró evitándolo al mismo tiempo" (MD 13-X-1921: 4).

Otras palabras pasan directamente del francés al español, adaptadas, como battre / batir; tirer (un coup) / tirar; tapis / tapiz; le train / el tren, por 'ritmo'; sonné / sonado: "Una inexplicable lentitud le impidió acabar con el francés, que andaba «sonado»" (E, 15-V-1927: 2). Mundo Deportivo solía introducir galicismos crudos que no tuvieron éxito al tener fácil adaptación o traducción. Es el caso de corps à corps, arrêts, defaite, matinée, soirée y alguna otra que utilizó en las dos primeras décadas, como la expresiva poulain, 'potro', para referirse a los novatos que empezaban a destacar: "ya que están seguros que su poulain vengará al tan cacareado Jim Jeffries, derrotando, aplastando a Jhonson en toda la línea” (MD 25-VI-1914: 5). Es un préstamo semántico, pues la traducción de pupilo, que terminaría triunfando en el lenguaje deportivo en la segunda mitad del siglo, toma muy bien la segunda acepción

${ }^{6}$ En su tratado sobre la esgrima estrenado el siglo XX, Carbonell (1900) recoge ya muchas de estas palabras como léxico propio de este deporte que en seguida pasarían al boxeo: asalto, ataque, contra, cruzado, cubrirse, cuerpo a cuerpo, doblar, finta, golpe, guardia, guardia alta, guardia baja, abrir la guardia, parar, tocado, uno-dos, etc. 
francesa de 'patrocinado' o 'protegido', aunque ya por entonces también se usaba en alguna ocasión: "Descamps, «manager» o director de Carpentier, saltó sobre el «ring», y cogiendo de la cintura a su pupilo, llevóselo a un rincón, obligándole a abandonar" (ABC 28-VI-1912: 3).

Otros préstamos de este tipo vienen con verbos como encajar, de encaisser, con el sentido de 'aguantar' y 'asumir los golpes', que se hace habitual en el segundo cuarto del siglo pasado: "Añade que el «punch» de Honey es ineficaz en la categoría y que duda mucho que Paulino, que encajó con la sonrisa en los labios los «swings» de Hansen cuando este podía combatir a distancia..." (E 2-IV-1927: 1), y librar, de livrer, por realizar o entablar combate: “Tiempo atrás, Marqués y Plasmn libraron un combate de parecida ferocidad, del cual salió vencedor Plasín” (MD 17-III-1921: 4). También se calcaron, entre otras, expresiones como hors combat / fuera de combate o jeu de jambes / juego de piernas: "Causó excelente expresión su forma ágil, su excelente juego de piernas y su vista y rapidez de desplazamiento” (MD 25-V-1923: 3).

\section{CONCLUSIONES}

En el primer tercio del siglo XX, época del inicio y de los primeros pasos de la práctica del boxeo en España, es cuando se forma en gran medida la lengua de este deporte, en el que anglicismos y galicismos tienen una importante presencia y contribuyen a crear numerosos neologismos. Hay distintos niveles neológicos:

1. Préstamos directos o intonsos: K.O., manager, jab, uppercut, clinch, ringside, sparring, out, break, welter y crouch, del inglés. Challenger, puncheur, footing, forcing, adaptaciones francesas del inglés.

2. Préstamos adaptados: boxeo (boxing); púgil (pugilist); nocaut y noquear (knock out); segundo / second; grogui (groggy); gongo (gong); finta (feint), etc., del inglés. Coquilla (coquille); esquiva (esquive); contra (contre); tapiz (tapis), promotor (promoteur), etc., del francés.

3. Préstamos directos que se alternan con traducciones o creaciones propias: ring / cuadrilátero; round / asalto; punch / pegada; speaker / locutor; infighting / cuerpo a cuerpo; swing / bolea; hook / gancho; cross / cruzado y algunos más, en inglés. Match / combate; crochet / gancho; punching ball / balón; sonné/ sonado, etc., en francés.

4. Antiguos préstamos extranjeros, luego sustituidos por calcos y traducciones: contragolpear (counterpunch); árbitro (referee), en inglés. Pupilo (poulain) o saco (punching bag), del francés.

5. Calcos desde el inicio: uno-dos (one-two), pesos mosca, pesos pluma, pesos ligeros, pesos medios, etc., del inglés. Pesos gallo, pesos pesados, cuerpo a cuerpo, 
fuera de combate y otros, del francés.

6. Préstamos directos de uso efímero: side step, reach, rush, fould, match macker, punch drunk, del inglés. Arrêts, matinée, soirée, etc., del francés.

Así pues, vista la decantación a que se sometió la primera avalancha de extranjerismos ingleses y franceses, hay que concluir que los años posteriores a la guerra civil son los de consolidación del léxico fundamental del boxeo en castellano, para lo cual no se hizo sino traducir, cribar y adaptar aquella entrada a veces arbitraria de la primera época.

Hoy, la Real Academia Española o la Fundación del Español Urgente (Fundéu) siguen velando por la pureza y adaptación de ese léxico y proponen soluciones acordes con la norma castellana, y cada vez más términos pasan a los diccionarios debido a su presencia en la lengua coloquial, pues son capaces de crear derivados y locuciones y desdoblar su primitivo significado en otros figurados, como sucede con noquear y arrojar la toalla, por poner solo dos ejemplos.

\section{REFERENCIAS BIBLIOGRÁFICAS}

\section{CORPUS DOCUMENTAL}

(ABC). ABC (1903-2017). Incluye (ByN) Blanco y Negro (1891-2000). hemeroteca.abc.es

(MD). El Mundo Deportivo (1906-1999). www.mundodeportivo.com/hemeroteca. (E). Excelsior (1927). htpp://www.liburuklik.euskadi.net/.

\section{BIBLIOGRAFÍA}

AGULLÓ, Recaredo (2003): Diccionario Español de términos deportivos. Madrid: Espasa Calpe.

ALFARO, Ricardo J. (1970): Diccionario de anglicismos. Madrid: Gredos.

CARDELLS MARTÍ, Francesc y Carlos GENER GALBIS (2004): “Historia, lenguaje y hecho deportivo: consideraciones a propósito del boxeo". Comunicación y Estudios Universitarios. 12, 41-50.

CARBONELL, Pedro (1900): Teoría y práctica de la esgrima. Barcelona: Sucesores de Rivadeneira.

CASTAÑÓN RODRÍGUEZ, Jesús (2004): Diccionario terminológico del deporte. 
Gijón: Trea.

CASTAÑÓN RODRÍGUEZ, Jesús, Emilio Tomás GARCÍA MOLINA y Edmundo LOZA OLAVE (2005): Términos deportivos en el habla cotidiana. Logroño: Universidad de La Rioja.

CEBRIÁN BONÉ, José Luis (dir) (1989): La gran enciclopedia de los deportes olimpicos, Barcelona: Ediciones del Drac, vol. 3.

CURELL AGUILÀ, Clara (2009): Diccionario de galicismos del español peninsular contemporáneo. Strasbourg: Éditions de Linguistique et Philologie.

FERNÁNDEZ GARCÍA, Antonio (1972): Anglicismos en el español (1891-1936). Oviedo.

LORENZO, Emilio (1996): Anglicismos hispánicos. Madrid: Gredos.

MIQUELARENA, Jacinto (1934): Stadium (Notas de sport). Madrid: Espasa Calpe. PRATT, Chris (1980): El anglicismo en el español peninsular contemporáneo. Madrid: Gredos.

REAL ACADEMIA ESPAÑOLA (2014): Diccionario de la lengua española, 23. ${ }^{\circledR}$ ed. Madrid: Espasa.

RODRÍGUEZ FEU, Francisco (1987): El boxeo como deporte y profesión. Barcelona: Hispano Europea.

RODRÍGUEZ GONZÁLEZ, Félix (2012): “Anglicismos en el mundo del deporte: variación lingüística y sociolingüística”. Boletín de la Real Academia Española. Tomo XCII, Cuaderno CCCVI, 317-341.

- - (2013): "Pseudoanglicismos en español actual. Revisión crítica y tratamiento lexicográfico”. Revista Española de Lingüistica. 43/1, 123-169.

- - (dir.) y Antonio LILLO (1997): Nuevo diccionario de anglicismos. Madrid: Gredos.

SÁNCHEZ GARCÍA, Raúl (2009): "Boxeo y proceso de civilización en la sociedad española”. Apunts: Educación Física y Deportes. 96, 5-13.

VÁZQUEZ-AMADOR, María y M. Carmen LARIO DE OÑATE (2015): “Anglicismos en la prensa deportiva de principios del siglo XX y XXI: Estudio contrastivo". Epos. XXXI, 359-374.

VIADA, Antonio (1903): Manual de sport. Madrid: Adrian Romo editor, 685-705.

Fecha de recepción: 16 de octubre de 2017

Fecha de aceptación: 26 de diciembre de 2017 